Research Article

\title{
Study of the Construction Sequence of Overlapping Tunnels by the Shield Tunneling Method: A Case Study of the Longest Overlapping Tunnel in China
}

\author{
Zhiyong Yang $\mathbb{D}$, Yaowen Ding, Yusheng Jiang, Kuanda Fang, Linwei Dong,

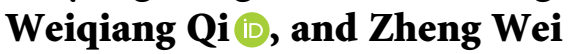 \\ School of Mechanics and Civil Engineering, China University of Mining \& Technology-Beijing, Beijing 100083, China \\ Correspondence should be addressed to Zhiyong Yang; yangzy1010@126.com
}

Received 8 January 2020; Revised 2 July 2020; Accepted 30 July 2020; Published 12 August 2020

Academic Editor: Venu G. M. Annamdas

Copyright ( 2020 Zhiyong Yang et al. This is an open access article distributed under the Creative Commons Attribution License, which permits unrestricted use, distribution, and reproduction in any medium, provided the original work is properly cited.

In this study, the reasonable construction sequence of an overlapping tunnel shield is investigated. Taking the overlapping tunnel of Tianjin Metro Line 5 as the background, a three-dimensional numerical model was established using Flac3D software to study the influence of the "first up and then down" and "first down and then up" construction sequences of the overlapping tunnel on the surface settlement, stratum displacement, lining radial stress, and displacement. The research results show that the shape and width of the final settlement tank on the ground under the two conditions are basically the same, and the difference between the maximum cumulative settlements is small. The accumulated ground settlement caused by the first up and then down construction sequence is $1.8 \mathrm{~mm}$ larger than that caused by the first down and then up sequence. The difference between the two working conditions on the vertical displacement of the stratum mainly occurs in the middle stratum of the upper and lower tunnels, and the proportion of the strata in the uplifted state and subsidence state is different. The construction sequence has little effect on the radial stress on the lining of the upper and lower tunnels. Under these two conditions, the excavation of the second tunnel causes a small change in the radial stress of the preceding tunnel lining, both within $4.2 \%$. Under the working condition of first down and then up, the construction of the second tunnel causes the lining of the preceding tunnel to rise by $7.2 \sim 9.2 \mathrm{~mm}$. Under the condition of first up and then down, the construction of the second tunnel causes the lining of the preceding tunnel to sink again by $9.1 \sim 10.4 \mathrm{~mm}$. By comparing the effects of the two working conditions on the stratum and the tunnel lining, it is recommended that the construction be carried out in the order of first down and then up.

\section{Introduction}

Overlapping tunnels refer to the two tunnels arranged vertically in the vertical direction. Compared with the two tunnels that are arranged in the horizontal direction, overlapping tunnels have a smaller influence area on the ground and stratum. As shown in Figure 1, when the road above the shield tunnel is narrow and there are structures such as buildings on both sides of the road, the use of horizontal parallel tunnels will greatly affect the structures, but the use of overlapping tunnels avoids this problem.

Compared to parallel tunnels, the cumulative ground settlement and stratum deformation caused by overlapping tunnel construction is greater, and the second tunnel shield construction inevitably has a greater impact on the preceding tunnel structure. The effect of overlapping tunnels on the surface ground settlement, stratum displacement, structure stress, and structure displacement is also different in different construction sequences. Therefore, it is necessary to study the strata and structure deformation caused by different construction sequences of overlapping tunnels.

At present, the most commonly used method to study this problem is to use finite element software for numerical analysis research. Dias et al. [1] conducted numerical simulations using Flac3D software to study the effects of overlapping tunnel construction in soft soil on the normal 


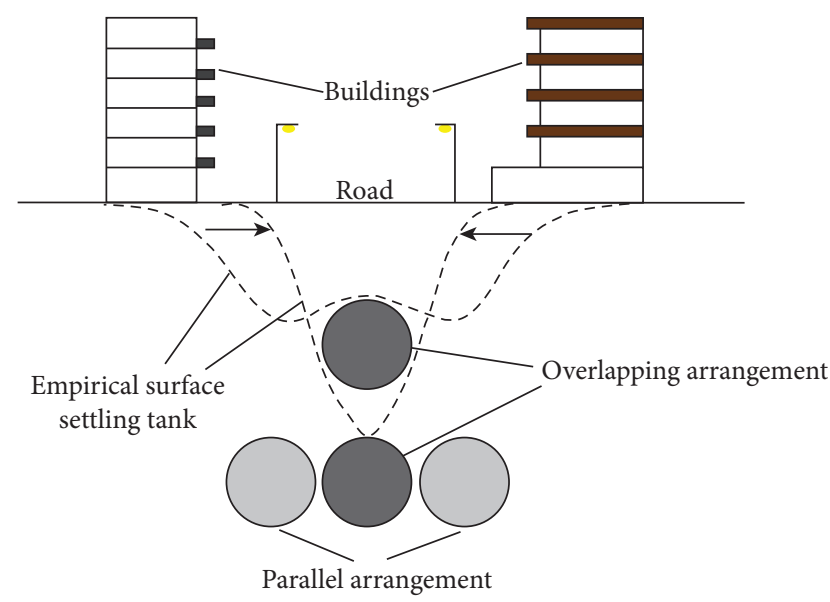

FIGURE 1: Comparison of influence area of different arrangement.

displacement, normal force, longitudinal force, and bending moment of the tunnel lining, as well as the surface ground settlement and horizontal displacement. Dias and Kastner [2] compared the actual engineering shield tunneling process with several numerical simulations and concluded that three-dimensional numerical analysis, which more directly considers the physical properties of the problem, is the most accurate method to simulate tunnel excavation, but it still has the disadvantage of not fully considering the shield tunneling process. Liu et al. [3], taking the overlapping tunnel of Shenzhen Metro Line 5 as the background, used numerical methods to study the effects of excavation stress release and seepage on the tunnel structure and soil deformation at different stages of the overlapping tunnel construction. Hasanpour et al. [4] used Flac3D to establish a numerical model, considered the interaction between the rock mass and the shield shell, lining and backfilling, and studied the impact of the propulsion rate and propulsion on the possibility of jamming during shield advance. Zheng et al. [5] used the finite element software Abaqus to perform a three-dimensional simulation, studied the stress history and pore pressure of the soil around the tunnel, and discussed the deformation mechanism of the tunnel. Chakeri et al. [6] and Naseem et al. [7] studied the stress distribution and settlement of tunnel construction under different layout conditions through finite element analysis. Liu et al. [8] proposed an analysis method to study the impact of new tunnels on existing tunnels, verified the method through the finite element method, and studied the mechanical behavior of existing tunnels under different parameters. Gharehdash and Barzegar [9] and Yan et al. [10] studied the dynamic response of shield tunneling using a three-dimensional numerical model.

The model test is also an effective method to study the stratum displacement and lining deformation caused by overlapping tunnel construction. Zhang et al. [11] studied the longitudinal displacement of the first tunnel directly below caused by the construction of the upper tunnel (after tunneling) by combining a three-dimensional finite element numerical calculation and an indoor centrifugal model test. Xie et al. [12] studied the variation rules of the displacement and internal force of existing tunnels during the construction of overlapping shield tunnels in soft soil due to different excavation sequences and different advance speeds by designing four sets of model tests. Choi and Lee [13] studied the effects of the tunnel spacing and earth pressure coefficient on existing tunnels through experimental model tests.

Some scholars have studied the lining deformation and stratum displacement of overlapping tunnels based on theory. Yang et al. [14] established a mathematical model of tunnel-induced surface motion based on the random medium theory and studied the convergence and influence angle using the back analysis method. Zhang et al. [15] proposed a calculation method for the surrounding strata deformation caused by the excavation of a double-line shield tunnel under any arrangement based on Schwarz's alternating method of displacement control and the complex variable function theory, combined with the boundary conditions of convergent deformation of the tunnel periphery. Based on highway tunnel design specifications, Gong et al. [16] established the surrounding rock pressure analysis model and calculation method of shallow buried tunnels with small net distances considering the construction sequence of double tunnels.

This study takes the longest overlapping tunnel in China as the overlapping tunnel between Chenglindao Station and Jintanglu Station of Tianjin Metro Line 5 (referred to as Cheng-jin section) as the background. The 500th to 550th sections of the tunnel are used as the research sections, and a $3 \mathrm{D}$ numerical model, built using Flac3D numerical simulation software, analyzes the effects of different shield construction sequences on the surface ground settlement, stratum displacement, and tunnel lining. Finally, the calculation results are verified using field monitoring data.

\section{Project Profile}

The Cheng-jin section has a total length of $1348.2 \mathrm{~m}$. The upper and lower sections of the tunnel completely overlap. The upper-line tunnel crosses the stratum with a completely broken floury clay layer. The lower-line tunnel crosses the stratum mainly with silty clay layers. The partial sections through the stratum are silty clay and a mixed formation of sandy silt. Two HERIK earth pressure balance shield tunnel boring machines (EPB-TBMs), each with a diameter of $6410 \mathrm{~mm}$, are used to construct the tunnels. The order of tunnel construction is first lower-line construction and then upper-line construction. The TBM excavation diameter is $6.43 \mathrm{~m}$, the segment external diameter is $6.2 \mathrm{~m}$, the segment internal diameter is $5.5 \mathrm{~m}$, the segment thickness is $0.35 \mathrm{~m}$, and the ring width is $1.2 \mathrm{~m}$.

The cross-sectional dimensions of the 500-550 ring research section of the line are shown in Figure 2. The leftline (upper-line) tunnel is buried at a depth of $13 \mathrm{~m}$, and the tunnel crosses the stratum of silty clay. The right-line (lowerline) tunnel has a buried depth of $24.2 \mathrm{~m}$, and the tunnel crosses the stratum as a mixed stratum of silty clay and sandy silt. Upper and lower tunnel spacings are $5 \mathrm{~m}$. 


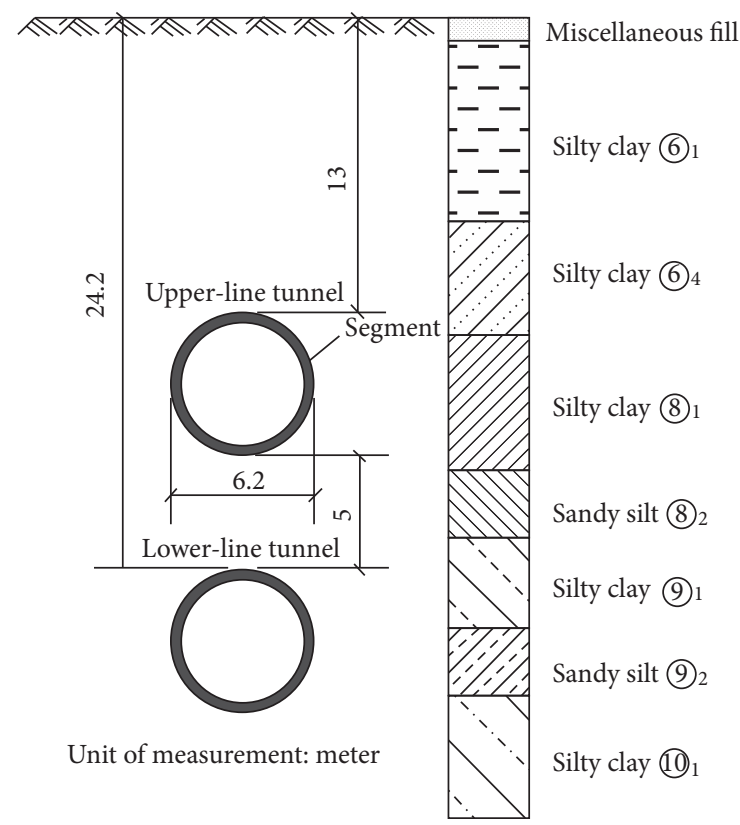

FIGURE 2: Study section profile.

\section{Measuring Point Arrangement}

In the actual project, to monitor the surface ground settlement, a monitoring section was set at the 525 ring, and 11 monitoring points were set, as shown in Figure 3.

\section{Numerical Modeling}

4.1. Modeling. A model of 500 to 550 rings of the line is established using Flac3D 5.0 numerical simulation software. The range of the soil is $50 \mathrm{~m}$ in the $X$-direction and $60 \mathrm{~m}$ in the $Y$-direction, and there are a total of 50 rings, which are $50 \mathrm{~m}$ in the $Z$-direction. The left (upper) tunnel is $13 \mathrm{~m}$ from the top of the model, the right (lower) tunnel is $24.2 \mathrm{~m}$ from the top of the model, and the distance between the upper and lower lines is $5 \mathrm{~m}$. The water table is located at $2 \mathrm{~m}$ below the ground surface. The segments and grouting layers are modeled according to actual working conditions. Ground load was not taken into account because the monitoring site was arranged in an open space and there were few structures. The model is shown in Figure 4.

The boundary conditions of the overall model are set as follows: the top surface of the model is a free surface, the bottom surface is subject to displacement constraints in the three directions $X, Y$, and $Z$, and the four sides are the constraints of the vertical normal displacement.

The Mohr-Coulomb constitutive model is used to calculate the soil in the simulation. The soil layer parameters are reasonably simplified by the weighted average of the soil layer thickness during modeling, as shown in Table 1. The current survey results of the project indicate that the groundwater flow here is only $1 \times 10^{-6} \mathrm{~cm} / \mathrm{s}$. Groundwater seepage is small in the research section, so the role of groundwater seepage is not considered. Both the segment and the grouting layer adopt an elastic model. The parameters are shown in Table 2.

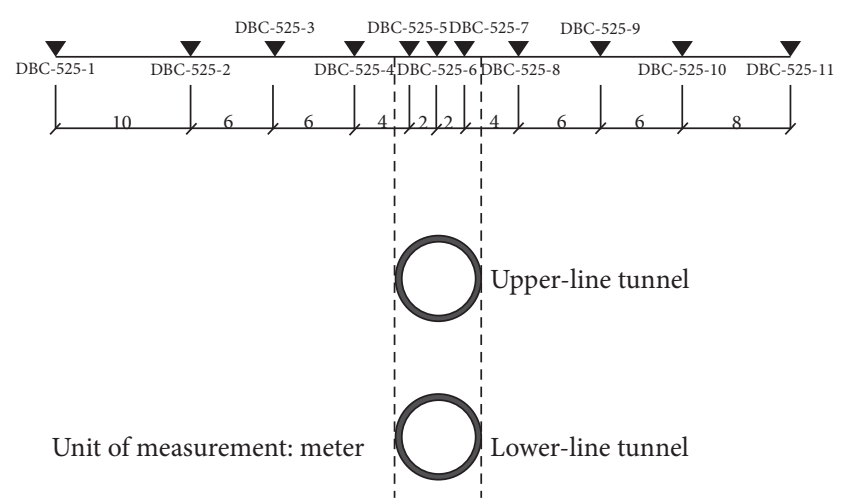

Figure 3: Monitoring section profile.

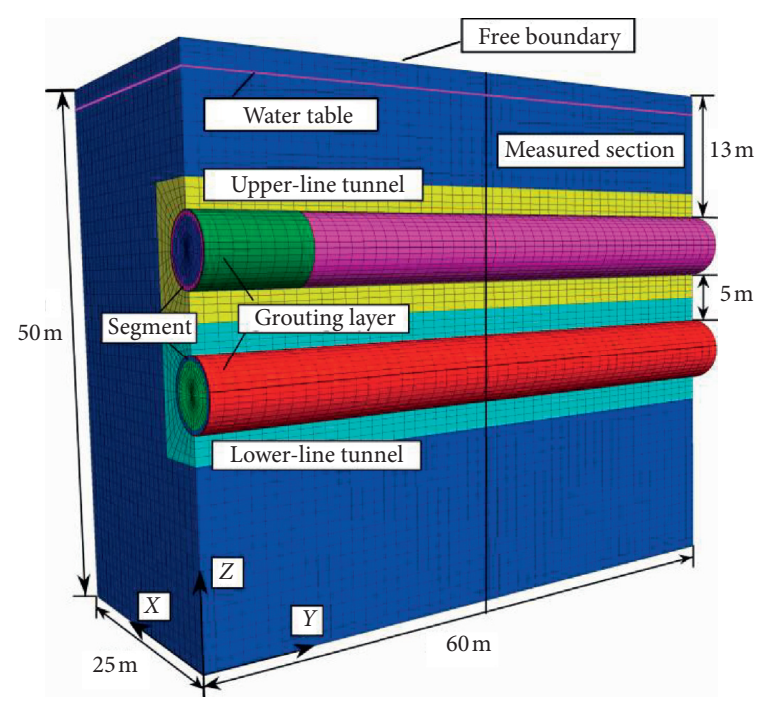

Figure 4: Half of the Flac3D numerical simulation model.

To study the surface settlement law of shield construction and the difference in the radial stress of the tunnel lining under two working conditions, as well as the disturbance of the construction of the second tunnel compared to the first tunnel and the stratum, the excavation sequence of "first down then up" and "first up then down" was used for the numerical simulation, and the target section (model $Y=30 \mathrm{~m}$ ) was taken as the research object.

4.2. Analysis of the Surface Settlement Law. When the shield constructs the lower-line tunnel first and then the upperline tunnel (first down and then up), the maximum ground settlement after the completion of the lower-line tunnel excavation is $-7.8 \mathrm{~mm}$. After the completion of the upper-line tunnel excavation, the maximum surface settlement is $-18 \mathrm{~mm}$, and the width of the settlement tank is approximately $48 \mathrm{~m}$. The settlement curve is shown in Figure 5.

When the shield constructs the upper-line tunnel first and then the lower-line tunnel (first up and then down), the maximum ground settlement after the completion of the lower-line tunnel excavation is $-12.1 \mathrm{~mm}$. After the completion of the upper-line tunnel excavation, the maximum 
TABLE 1: Soil mechanics parameters.

\begin{tabular}{|c|c|c|c|c|c|c|}
\hline $\begin{array}{l}\text { The soil } \\
\text { classification }\end{array}$ & $\begin{array}{l}\text { Thickness } h \\
(\mathrm{~m})\end{array}$ & $\begin{array}{l}\text { Unit weight } \gamma \\
\left(\mathrm{kN} / \mathrm{m}^{3}\right)\end{array}$ & $\begin{array}{c}\text { Cohesion } C \\
(\mathrm{kPa})\end{array}$ & $\begin{array}{c}\text { Internal friction angle } \\
\varphi\left({ }^{\circ}\right)\end{array}$ & $\begin{array}{l}\text { Compressive modulus Es } \\
(\mathrm{MPa})\end{array}$ & $\begin{array}{l}\text { Poisson's } \\
\text { ratio }\end{array}$ \\
\hline Plain fill & 1 & 19 & 8 & 12 & 4.2 & 0.35 \\
\hline Silty clay (6) 1 & 8 & 19.6 & 18.4 & 24.1 & 5.6 & 0.35 \\
\hline Silty clay (6) 4 & 5 & 19.4 & 18.2 & 23.9 & 6.5 & 0.3 \\
\hline Silty clay $8{ }_{1}$ & 6 & 20.4 & 21.5 & 19 & 5.5 & 0.3 \\
\hline Sandy silt (8) 2 & 3 & 21.5 & 4.6 & 36.2 & 14.4 & 0.3 \\
\hline Silty clay (9) 1 & 4 & 20.4 & 21.9 & 27.1 & 7.4 & 0.3 \\
\hline Sandy silt (9) 2 & 3 & 20.3 & 8.7 & 33.9 & 13.1 & 0.3 \\
\hline Silty clay (10) 1 & 20 & 22 & 39.4 & 23 & 7.6 & 0.3 \\
\hline
\end{tabular}

TABLE 2: Numerical simulation parameters.

\begin{tabular}{lcccc}
\hline & Poisson's ratios $\mu$ & Bulk modulus $K(\mathrm{MPa})$ & Shear modulus $G(\mathrm{MPa})$ & Density $\rho\left(\mathrm{kg} / \mathrm{m}^{3}\right)$ \\
\hline Segment & 0.17 & $1.57 \times 10^{4}$ & $1.295 \times 10^{4}$ & 2500 \\
Grouting layer & 0.2 & $4.57 \times 10^{2}$ & $3.96 \times 10^{2}$ & 2500 \\
\hline
\end{tabular}

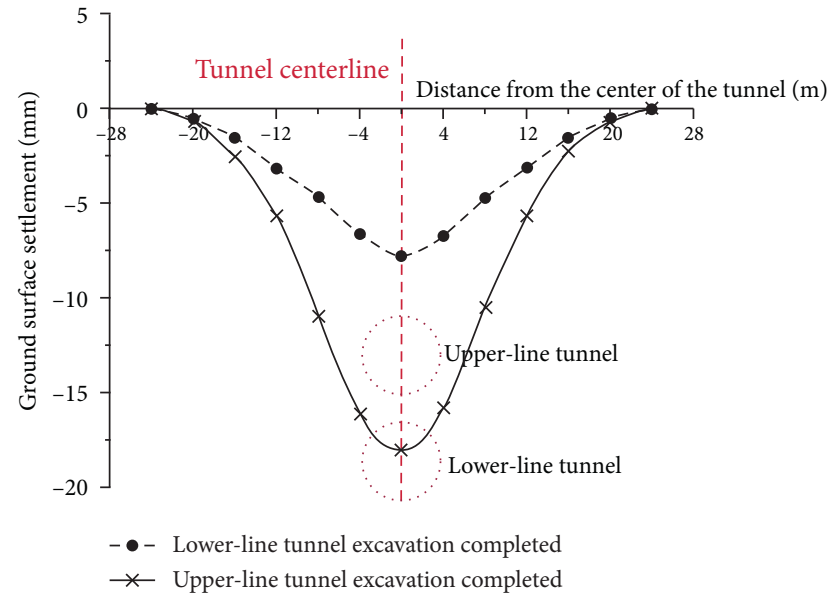

Figure 5: First down and then up construction sequence surface settlement curve.

surface settlement is $-19.8 \mathrm{~mm}$, and the width of the settlement tank is approximately $48 \mathrm{~m}$. The settlement curve is shown in Figure 6.

Under the two working conditions, the shape and width of the final settlement tank on the ground surface are basically the same, and the difference between the maximum cumulative settlement amounts is very small. The accumulated ground settlement caused by the first up and then down construction sequence is $1.8 \mathrm{~mm}$ larger than that caused by the construction of the first down and then up.

4.3. Stratum Vertical Displacement Analysis. Figures 7(a) and 7(b) show the contour maps of the vertical displacement of the stratum during construction of the first down and then up sequence.

Figures 8(a) and 8(b) show the contour maps of the vertical displacement of the stratum during construction in the sequence of first up and then down.

The stratum is divided into three parts for the vertical displacement analysis as follows.

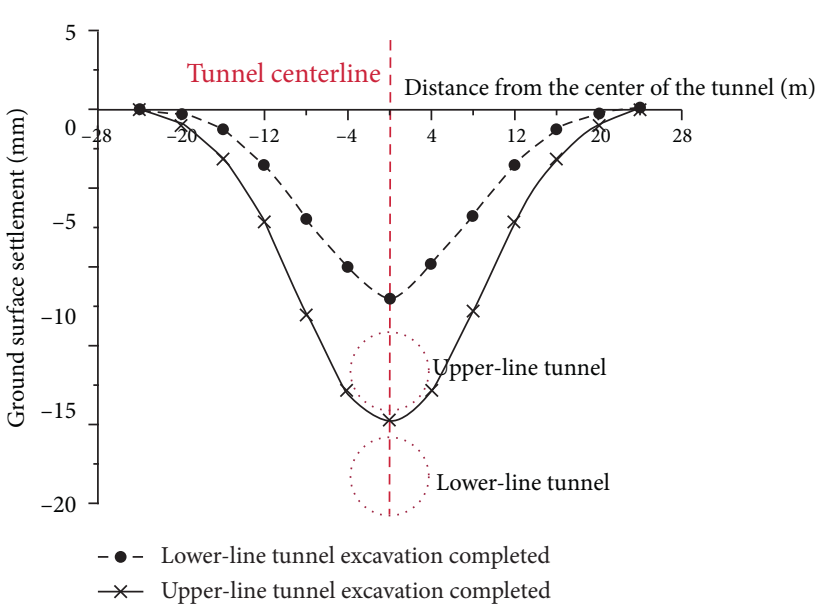

Figure 6: First up and then down construction sequence surface settlement curve.

4.3.1. Strata below the Lower-Line Tunnel. During the sequence of first down and then up construction, the lower strata uplift occurs after the lower-line tunnel excavation is completed. After the completion of the upper-line tunnel excavation, further uplift occurred in the lower strata of the lower-line tunnel.

During the construction sequence of first up and then down, after the upper-line tunnel excavation is completed, the lower-line tunnel has not yet been excavated, and the lower strata of the lower-line tunnel are also uplifted. After the completion of the lower-line tunnel excavation, a large uplift occurred in the lower strata.

In both cases, the construction of the second tunnel will destroy the displacement field formed after the first tunnel is constructed, and the final uplift amount and range of the lower strata of the lower-line tunnel are basically the same.

4.3.2. Middle Stratum of the Upper and Lower Tunnels. During the construction of the first down and then up sequence, the middle stratum of the upper and lower tunnels will sink to a certain extent after the completion of the 


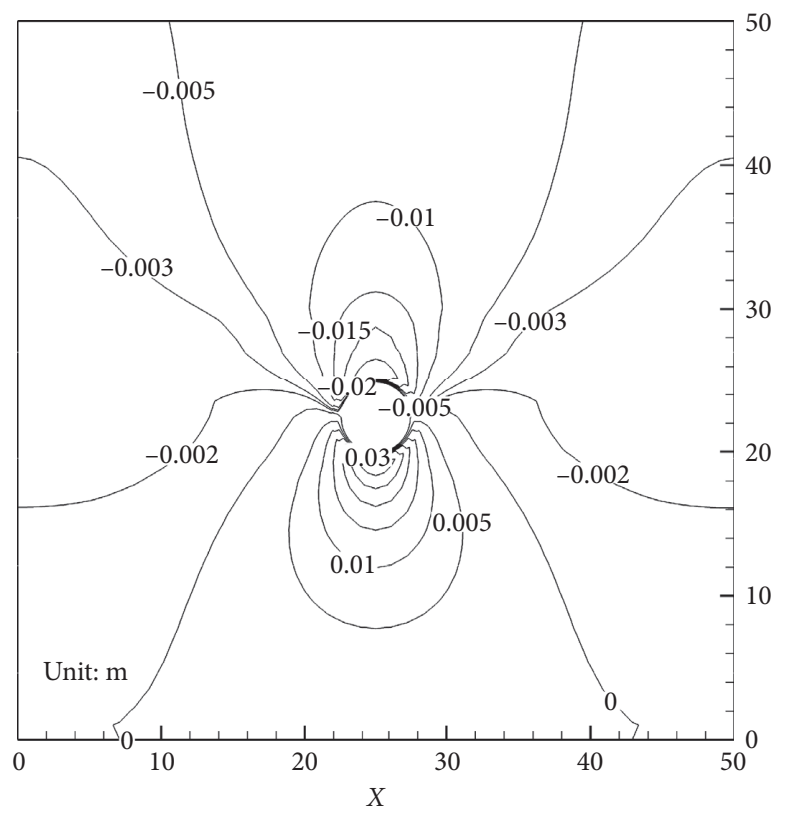

(a)

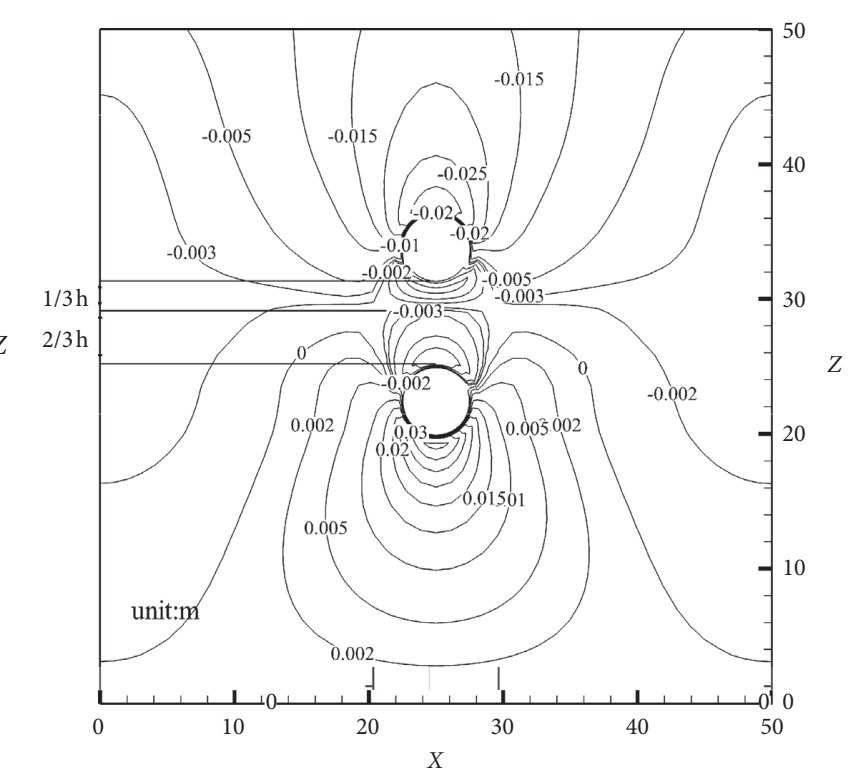

(b)

Figure 7: (a) Contour map of the vertical displacement of the stratum after excavation of the lower tunnel. (b) Contour map of the vertical displacement of the stratum after excavation of the upper tunnel (h: the distance between the upper and lower tunnels).

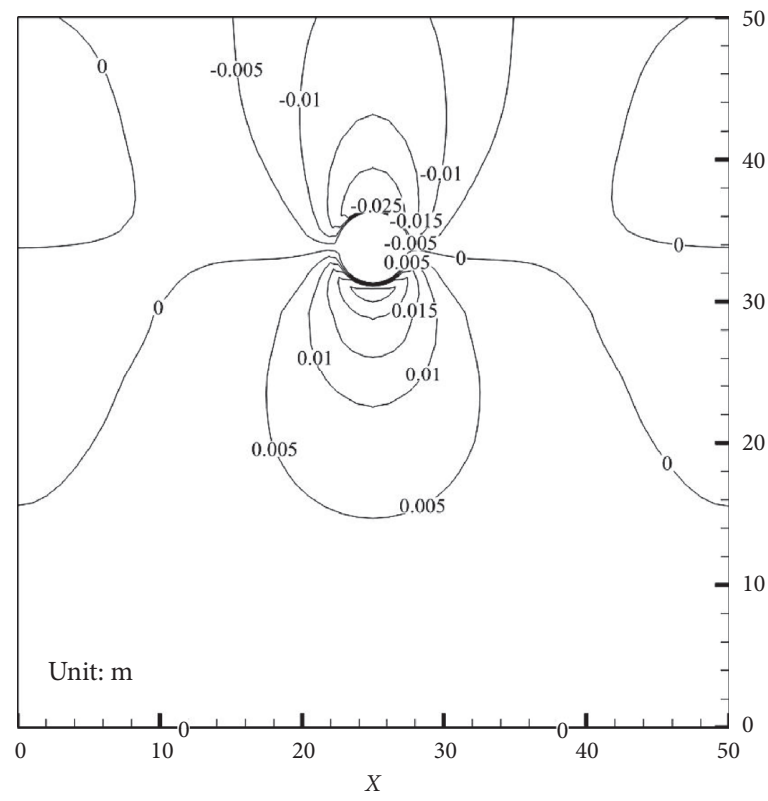

(a)

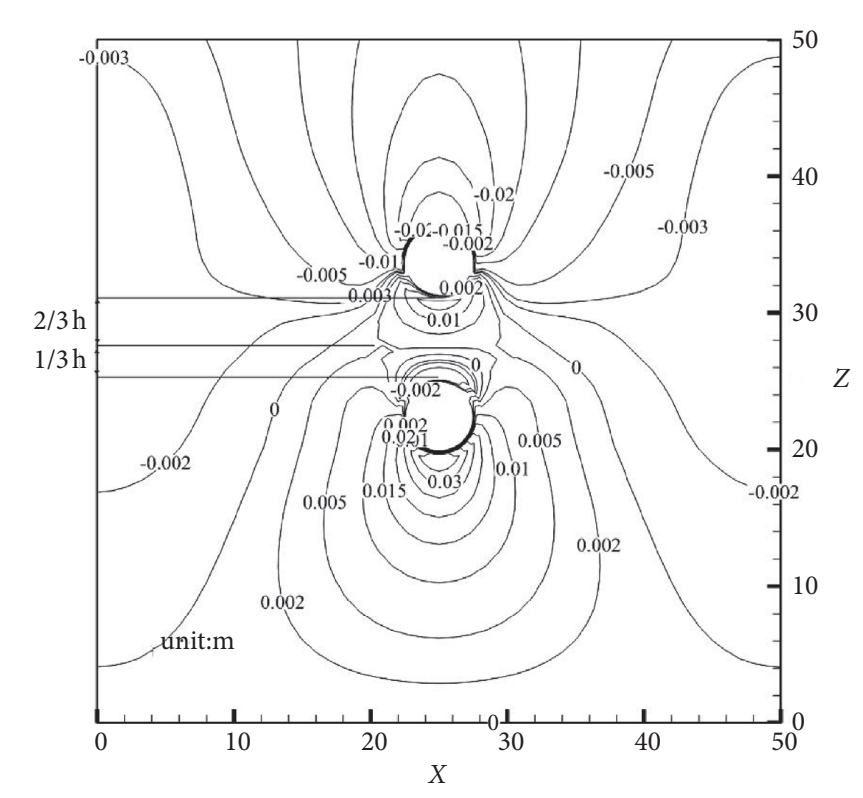

(b)

Figure 8: (a) Contour map of the vertical displacement of the stratum after the excavation of the upper tunnel. (b) Contour map of the vertical displacement of the stratum after the excavation of the lower tunnel (h: the distance between the upper and lower tunnels).

lower-line tunnel excavation, and the stratum will rise after the completion of the upper-line tunnel excavation. Additionally, some strata close to the upper-line tunnel will change from a sinking state to an uplift state. Finally, the stratum range in the uplift state is approximately $1 / 3 \mathrm{~h}(\mathrm{~h}$ : net distance between the upper-line and lower-line tunnels) below the bottom of the upper-line tunnel, and the stratum range in the sinking state is approximately $2 / 3 \mathrm{~h}$ above the top of the lower-line tunnel.

During the construction in the sequence of first up and then down, a certain degree of uplift occurred in the middle stratum of the upper- and lower-line tunnel after the upperline tunnel excavation was completed, the stratum sank after the lower-line tunnel excavation, and some strata close to the 
lower-line tunnel changed from an uplift state to a sinking state. Finally, the stratum range of the sinking state is approximately $1 / 3 \mathrm{~h}$ above the top of the lower-line tunnel, and the stratum range of the uplift state is approximately $2 / 3 \mathrm{~h}$ below the bottom of the upper-line tunnel.

4.3.3. Strata above the Upper-Line Tunnel. Under the two working conditions, the stratum sinks after the construction of the first tunnel and the excavation of the second tunnel causes the stratum to sink further. Under both conditions, the construction of the second tunnel will destroy the displacement field formed after the construction of the first tunnel, and the subsidence amount and the final settlement range of the upper strata of the upper-line tunnel are basically the same.

From the vertical displacement analysis of the stratum, it can be seen that the difference in the vertical displacement of the stratum under the two working conditions mainly occurs in the stratum between the upper and lower tunnels, which is mainly reflected in the different proportions of the strata in the uplift state and the subsidence state.

\subsection{Radial Stress Analysis of the Lining}

4.4.1. Radial Stress of the Upper and Lower Tunnel Lining. The radial stress of the upper-line tunnel after the completion of double-line construction under two working conditions is shown in Figure 9. Under the two working conditions, the radial stress of the tunnel lining is the minimum at the arch waist and the maximum at both sides of the arch bottom. The radial stress of the upper half-circle lining is basically the same, and the radial stress of the lower half-circle lining of the first down and then up sequence is lower by approximately $0.01 \sim 0.02 \mathrm{MPa}$ than that of the first up and then down sequence. The decrease rate is $4.5 \% \sim 8 \%$.

The radial stress of the lower-line tunnel after the completion of double-line construction under two working conditions is shown in Figure 10. Under the two conditions, the radial stress of the tunnel lining is the minimum at the arch waist and the maximum at both sides of the arch bottom. The radial stress of the first up and then down sequence of the lining near the vault and the bottom of the arch is lower than that of the first down and then up sequence, and the difference is more obvious near the vault. The first up and then down working conditions are 0.04 0.07 MPa less than the first down and then up working conditions. The decrease rate is $8 \% \sim 10 \%$, but the radial stress of the lining of the first down sequence and then up near the arch waist is lower than that of the first up and then down sequence.

The radial stresses of the upper and lower tunnel linings are all less different under the two conditions. In addition, the project adopts the reinforced concrete section lining of C50 (outer diameter $6.2 \mathrm{~m}$, thickness $0.35 \mathrm{~m}$, and ring width $1.2 \mathrm{~m}$ ), and the strength and stiffness of the segment are large. A 0.01 0.07 MPa variation in the radial stress has little effect on the internal force of the lining.

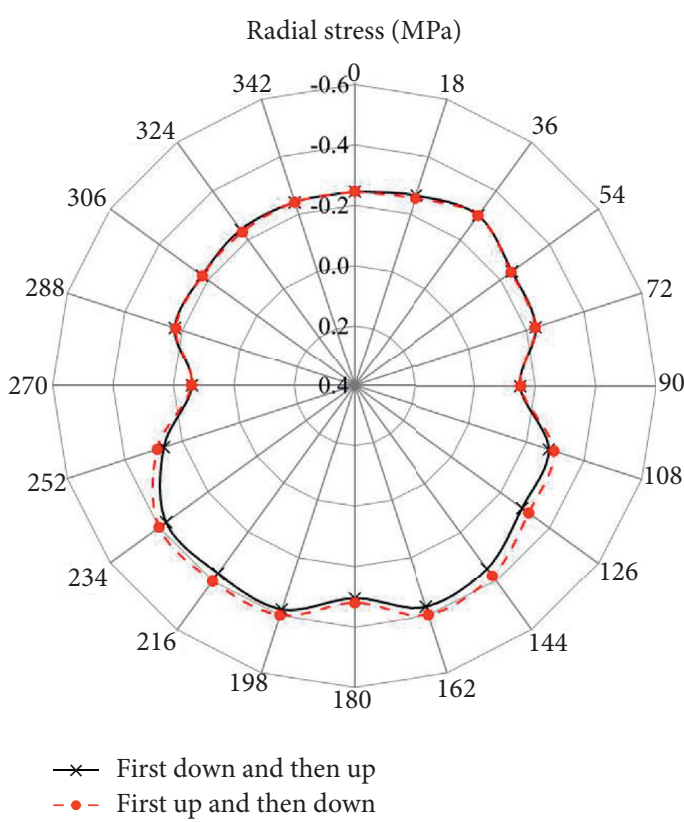

Figure 9: Radial stress of the upper tunnel lining.

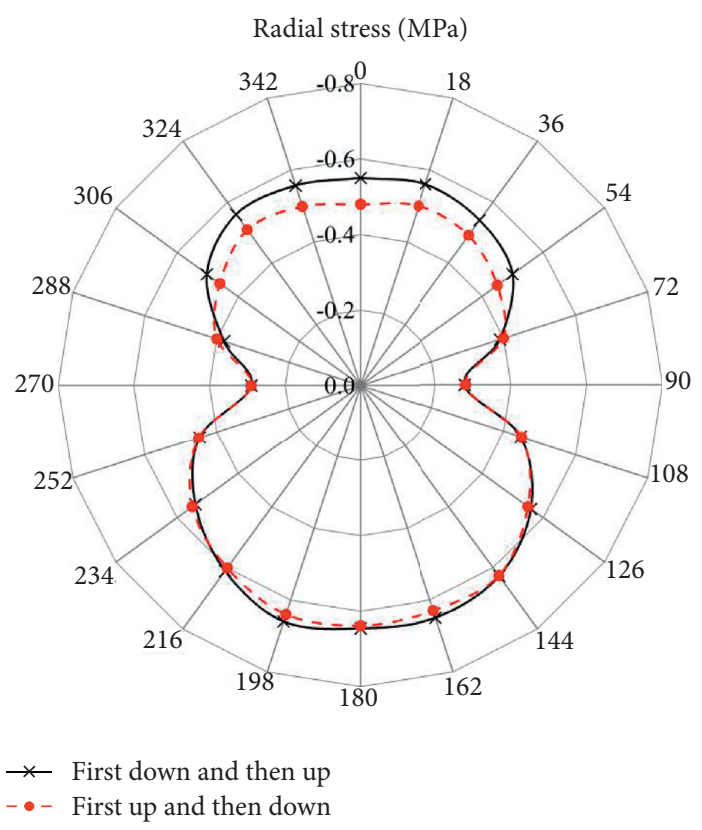

FIGURE 10: Radial stress of the lower tunnel lining.

4.4.2. The Influence of the Second Tunnel on the Radial Stress of the First Tunnel. Under the working condition of first down and then up, the radial stress of the lining after the excavation of the first lower-line tunnel is set to 0 . After the excavation of the upper-line tunnel is completed, the change in the radial stress of the lower-line tunnel lining is shown in Figure 11 (a positive value means increase; negative value means decrease). After the construction of the upper-line tunnel, the radial stress on the lining of the vault and the bottom of the arch decreases, and the change in the radial stress of the lining at the bottom of the arch is slightly greater than that at the top of the arch, with a maximum reduction 


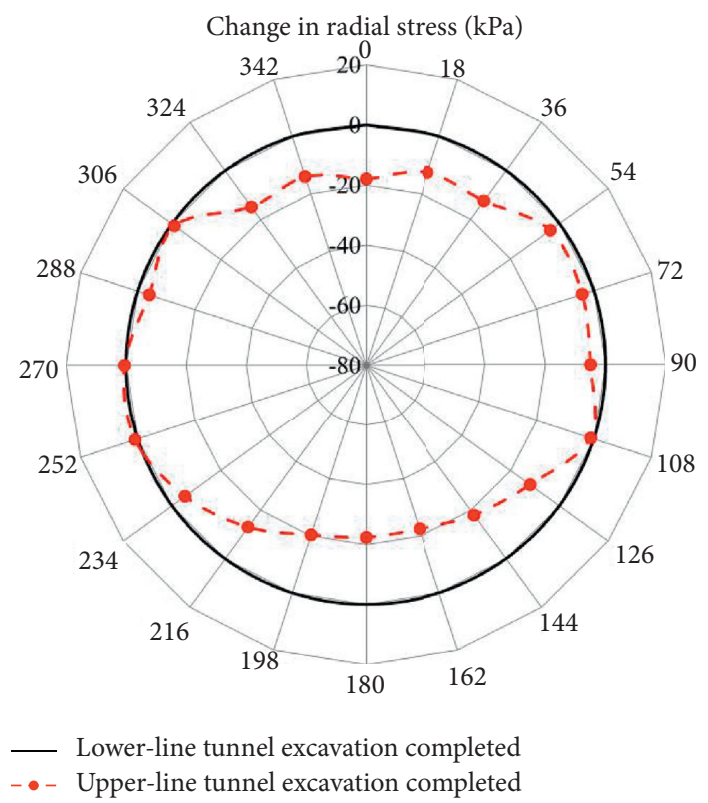

Figure 11: Change in the radial stress.

of $22.4 \mathrm{kPa}$, a decrease of $3.4 \%$. However, the change in the radial stress of the lining at both sides of the arch is small.

Under the working condition of first up and then down, the radial stress of the lining after the excavation of the first lower-line tunnel is set to 0 . After the construction of the lower tunnel is completed, the change in the radial stress in the lining of the upper-line tunnel is shown in Figure 12 (positive value means increase; negative value means decrease). After the construction of the lower-line tunnel, the radial stress on the lining of the vault and the bottom of the upper-line tunnel is decreased, and the radial stress change in the lining at the arch bottom is slightly larger than the radial stress change of the lining at the arch top. The maximum reduction is $8 \mathrm{kPa}$, which is $2.4 \%$. The radial stress of the lining near the arch waist on both sides is increased, and the maximum increase is $11.5 \mathrm{kPa}$, an increase of $4.2 \%$.

In both cases, the excavation of the second tunnel causes a small change in the radial stress of the first tunnel lining, both within $4.2 \%$. Therefore, under different construction orders, the shield tunnel construction of the second tunnel has little effect on the stress of the first tunnel lining.

\subsection{Analysis of the Influence of the Second Tunnel on the Lining} Deformation of the First Tunnel. Under the condition of first down and then up, the deformation of the lining of the lower tunnel after the upper tunnel is constructed is shown in Figure 13. The deformation characteristics of the lining are mainly floating. The maximum floating amounts of the lining at the arch top and arch bottom are $9.2 \mathrm{~mm}$ and $7.2 \mathrm{~mm}$, respectively. The lining has a certain degree of ovalization due to the symmetry of the excavated tunnel and the model, and the horizontal deformation of the lining is symmetrically distributed with minimal changes.

Under the condition of first up and then down, the deformation of the lining of the upper-line tunnel after the

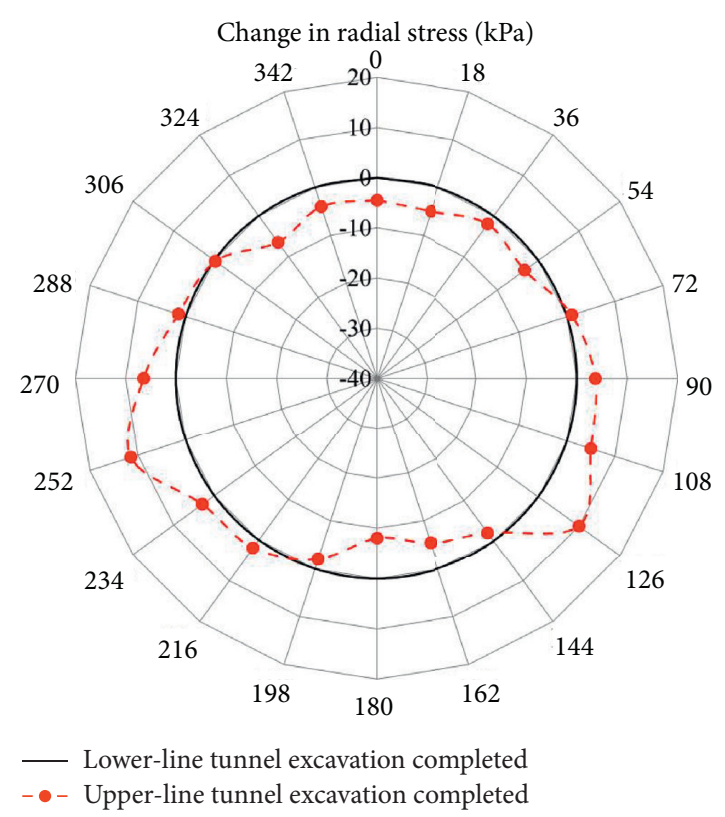

FIgURE 12: Change in the radial stress.

construction of the lower tunnel is shown in Figure 14. The deformation characteristics of the lining are mainly sinking. The maximum sinking amount of the lining at the arch top is $-9.1 \mathrm{~mm}$, and the maximum sinking amount of the lining at the arch bottom is $-10.4 \mathrm{~mm}$. The lining has a certain degree of ovalization, similar to the working conditions of first down and then up, and the horizontal deformation of the lining is symmetrically distributed with minimal changes.

\section{Verification with Measured Data}

The date when the right (lower-line) shield reached the monitoring section in the Cheng-jin section was August 26, 


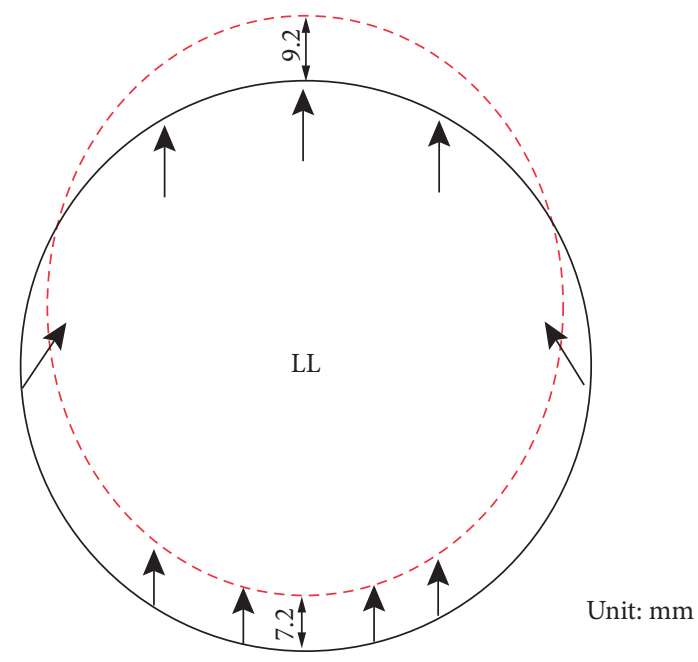

- Lower-line tunnel excavation completed

- - Upper-line tunnel excavation completed

Figure 13: The lining deformation of the lower-line tunnel.

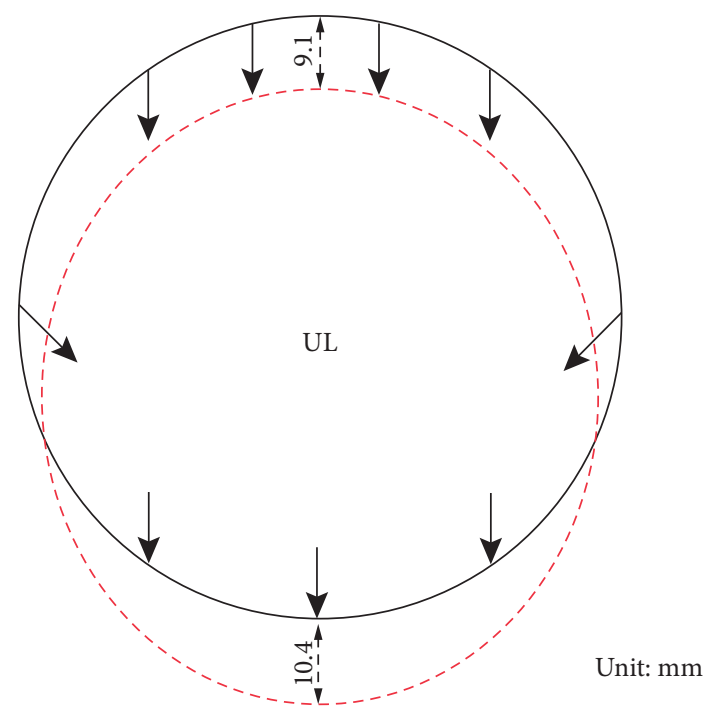

_ Lower-line tunnel excavation completed

- - - Upper-line tunnel excavation completed

Figure 14: The lining deformation of the upper-line tunnel.

2015, and the date when the left (upper-line) shield reached the monitoring section was August 9, 2016. The construction of the shield tunnel through the monitoring section is carried out strictly in accordance with the preset plan, and the monitoring curve is used to generate the settlement curve, as shown in Figure 15.

Through the analysis of the field monitoring data, the following can be known:

In the Cheng-jin section, the maximum settlement value of the ground surface after construction of the right-line (lower-line) tunnel is $-5 \mathrm{~mm}$, and the maximum settlement value of the surface after construction of the left-line (upperline) tunnel is $-17.2 \mathrm{~mm}$. During the construction process, the maximum settlement point on the ground surface is

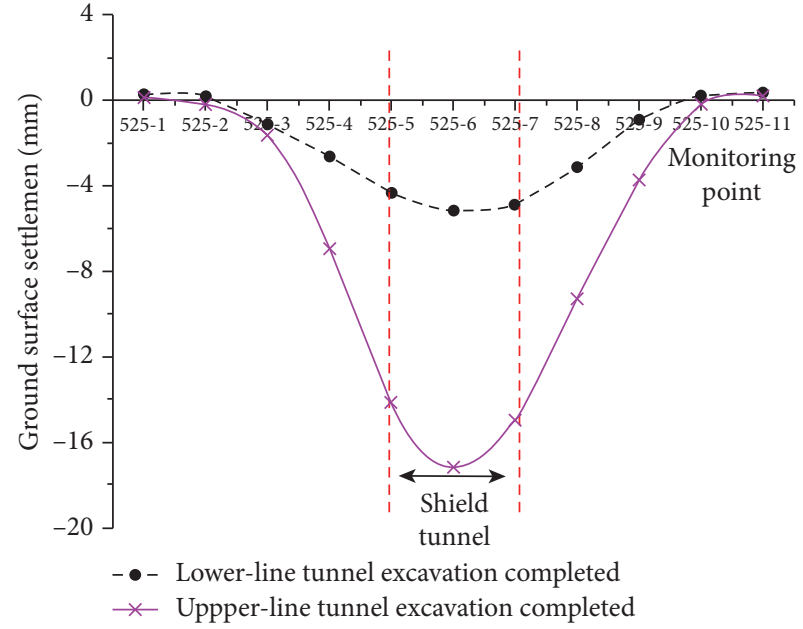

FIGURE 15: Surface subsidence curve of the actual engineering.

always directly above the tunnel axis, and the width of the settlement tank and maximum cumulative settlement is basically consistent with the numerical simulation results. The settlement curve extracted by preanalysis through numerical simulation is basically in line with the actual construction situation, which can predict the law of the surface settlement and stratum displacement under different working conditions.

\section{Conclusions}

Taking the overlapping tunnel of the Cheng-jin section of Tianjin Metro Line 5 as the background, the ground subsidence law and the vertical displacements of the stratum and radial stress and deformation of the tunnel lining under different construction sequences are studied through numerical simulation, which is demonstrated with field-measured data. The following conclusions are obtained:

(1) Under the construction sequences of first down and then up and first up and then down, the shape and width of the final settlement trough on the surface are basically the same, and the change in the maximum cumulative settlement is very small. The cumulative settlement caused by the construction of first up and then down is $1.8 \mathrm{~mm}$ larger than that caused by the construction sequence of first down and then up.

(2) The difference between the effects of the two conditions on the vertical displacement of the stratum mainly occurs in the middle stratum of the upper and lower tunnels, and the strata in the states of uplift and sinking are different. Under the condition of first down and then up, the stratum range of the uplift state is approximately $1 / 3 \mathrm{~h}$ below the bottom of the upper-line tunnel, and the stratum range of the subsidence state is approximately $2 / 3 \mathrm{~h}$ above the top of the lower-line tunnel. Under the condition of first up and then down, the stratum range of the sinking 
state is approximately $1 / 3 \mathrm{~h}$ above the top of the lower-line tunnel, and the stratum range of the uplift state is approximately $2 / 3 \mathrm{~h}$ below the bottom of the upper-line tunnel.

(3) The construction sequence has little effect on the ultimate radial stress on the tunnel lining.

(4) Under the two working conditions, the change in the radial stress of the first tunnel lining caused by the excavation of the second tunnel is very small, both within $4.2 \%$. Therefore, under different construction orders, the construction of the second tunnel shield has little influence on the stress of the first tunnel lining.

(5) Under the condition of first down and then up, the deformation mode of the lining of the first built tunnel caused by the second built tunnel is mainly upward floating, and the upward floating quantity is approximately $7.2 \sim 9.2 \mathrm{~mm}$. Under the condition of first up and then down, the deformation mode of the lining of the first tunnel caused by the second construction is mainly subsidence, and the subsidence is approximately $-9.1 \sim-10.4 \mathrm{~mm}$.

By comparing the surface settlement, stratum displacement, lining radial stress, and displacement under the two working conditions, it is suggested that the construction sequence of first down and then up is adopted because it causes less surface settlement and lining displacement.

\section{Data Availability}

The data used to support the findings of this study are available from the corresponding author upon request.

\section{Conflicts of Interest}

The authors declare that they have no conflicts of interest.

\section{Acknowledgments}

Financial support for this work provided by the National Natural Science Foundation of China (Grant no. U1261212) and Beijing Municipal Education Commission (Grant no. ZDZH20141141301) is gratefully acknowledged.

\section{References}

[1] D. Dias, N. A. Do, and P. Oreste, "Three-dimensional numerical simulation of mechanized twin stacked tunnels in soft ground," Journal of Zhejiang University-Science A, vol. 15, no. 11, pp. 896-913, 2014.

[2] D. Dias and R. Kastner, "Movements caused by the excavation of tunnels using face pressurized shields-analysis of monitoring and numerical modeling results," Engineering Geology, vol. 152, no. 1, pp. 17-25, 2013.

[3] W. Liu, X. W. Tang, P. L. Gan, and S. Savidis, "Soil deformation induced by overlapping tunneling in water-bearing strata," Chinese Journal of Geotechnical Engineering, vol. 35, no. 6, pp. 1055-1061, 2013.
[4] R. Hasanpour, J. Rostami, and B. Ünver, “3D finite difference model for simulation of double shield TBM tunneling in squeezing grounds," Tunnelling and Underground Space Technology, vol. 40, pp. 109-126, 2014.

[5] G. Zheng, T. Zhang, and Y. Diao, "Mechanism and countermeasures of preceding tunnel distortion induced by succeeding EPBS tunnelling in close proximity," Computers and Geotechnics, vol. 66, pp. 53-65, 2015.

[6] H. Chakeri, R. Hasanpour, M. A. Hindistan, and B. Ünver, "Analysis of interaction between tunnels in soft ground by 3D numerical modeling," Bulletin of Engineering Geology and the Environment, vol. 70, no. 3, pp. 439-448, 2010.

[7] A. Naseem, K. Schotte, B. de Pauw, and H. de Backer, "Ground settlements due to construction of triplet tunnels with different construction arrangements," Advances in Civil Engineering, vol. 2019, Article ID 8637837, 18 pages, 2019.

[8] X. Liu, Q. Fang, D. Zhang, and Z. Wang, "Behaviour of existing tunnel due to new tunnel construction below," Computers and Geotechnics, vol. 110, pp. 71-81, 2019.

[9] S. Gharehdash and M. Barzegar, "Numerical modeling of the dynamic behaviour of tunnel lining in shield tunneling," KSCE Journal of Civil Engineering, vol. 19, no. 6, pp. 16261636, 2015.

[10] Q.-X. Yan, L.-Y. Song, H. Chen, W.-Y. Chen, S.-Q. Ma, and W.-B. Yang, "Dynamic response of segment lining of overlapped shield tunnels under train-induced vibration loads," Arabian Journal for Science and Engineering, vol. 43, no. 10, pp. 5439-5455, 2018.

[11] H. J. Zhang, W. G. Qiu, J. M. Feng, Y. C. Zheng, and L. Gong, "Study of longitudinal mechanical behavior and countermeasure for neighborhood overlapped shield tunneling," Rock and Soil Mechanics, vol. 31, no. 11, pp. 3569-3573, 2010.

[12] X. Y. Xie, J. T. Niu, G. W. Yang, and C. Liu, "Model test for effects of construction of shield tunnelling in overlapping tunnels on existing tunnel," Chinese Journal of Rock Mechanics and Engineering, vol. 32, no. 10, pp. 2061-2069, 2013.

[13] J.-I. Choi and S.-W. Lee, "Influence of existing tunnel on mechanical behavior of new tunnel," KSCE Journal of Civil Engineering, vol. 14, no. 5, pp. 773-783, 2010.

[14] J. S. Yang, B. C. Liu, and M. C. Wang, "Modeling of tunnelinginduced ground surface movements using stochastic medium theory," Tunnelling and Underground Space Technology, vol. 19, no. 2, pp. 113-123, 2004.

[15] Z. G. Zhang, C. P. Zhang, and X. G. Xi, "Closed solutions to soil displacements induced by twin-tunnel excavation under different layout patterns," Chinese Journal of Geotechnical Engineering, vol. 41, no. 2, pp. 262-271, 2019.

[16] J. W. Gong, C. C. Xia, and X. W. Lei, "Analysis of field measurement and theoretical calculation on rock pressure in shallow-buried twin tunnels with small spacing," Chinese Journal of Rock Mechanics and Engineering, vol. 29, no. S2, pp. 4139-4145, 2010. 